\title{
Air-borne shape measurement of parabolic trough collector fields
}

Christoph Prahl, Marc Röger, and Christoph Hilgert

Citation: AIP Conference Proceedings 1850, 020013 (2017);

View online: https://doi.org/10.1063/1.4984338

View Table of Contents: http://aip.scitation.org/toc/apc/1850/1

Published by the American Institute of Physics

\section{Articles you may be interested in}

Parabolic trough receiver heat loss and optical efficiency round robin 2015/2016

AIP Conference Proceedings 1850, 020012 (2017); 10.1063/1.4984337

Full parabolic trough qualification from prototype to demonstration loop

AIP Conference Proceedings 1850, 020010 (2017); 10.1063/1.4984334

Shams 1 - Design and operational experiences of the $100 \mathrm{MW}-540^{\circ} \mathrm{C}$ CSP plant in Abu Dhabi

AIP Conference Proceedings 1850, 020001 (2017); 10.1063/1.4984325

Towards high efficiency heliostat fields

AIP Conference Proceedings 1850, 030001 (2017); 10.1063/1.4984344

Long-term heating to improve receiver performance

AIP Conference Proceedings 1850, 020006 (2017); 10.1063/1.4984330

Steady-state plant model to predict hydrogen levels in power plant components

AIP Conference Proceedings 1850, 020008 (2017); 10.1063/1.4984332 


\title{
Air-Borne Shape Measurement of Parabolic Trough Collector Fields
}

\author{
Christoph Prahl ${ }^{1, \text { a) }}$, Marc Röger ${ }^{1}$ and Christoph Hilgert ${ }^{1}$ \\ ${ }^{1}$ German Aerospace Center (DLR e.V.), Solar Research, PSA, 04200 Tabernas, Spain.

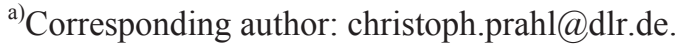

\begin{abstract}
The optical and thermal efficiency of parabolic trough collector solar fields is dependent on the performance and assembly accuracy of its components such as the concentrator and absorber. For the purpose of optical inspection/approval, yield analysis, localization of low performing areas, and optimization of the solar field, it is essential to create a complete view of the optical properties of the field. Existing optical measurement tools are based on ground based cameras, facing restriction concerning speed, volume and automation. QFly is an airborne qualification system which provides holistic and accurate information on geometrical, optical, and thermal properties of the entire solar field. It consists of an unmanned aerial vehicle, cameras and related software for flight path planning, data acquisition and evaluation. This article presents recent advances of the QFly measurement system and proposes a methodology on holistic qualification of the complete solar field with minimum impact on plant operation.
\end{abstract}

\section{INTRODUCTION}

The conversion from solar radiation to heat and electricity in CSP (Concentrating Solar Power) plants is characterized by the efficiency of a cascade of conversion steps like concentration and absorption of the solar radiation, heat losses to the environment during transport/storage of the thermal energy, and the performance of the power block. This article focuses on the optical and thermal performance, which is determined by the geometry and optical properties (reflectivity, transmissivity and absorptivity) of mirrors, glass envelope and absorber tubes, respectively. Development and implementation of CSP plants are always supported by specific measurement techniques in order to assess and improve performance. Optical measurement techniques using VIS (Visible Spectrum) or IR (Infrared) cameras are the first choice to determine the geometric, optical, and thermal properties. The application of UAVs (Unmanned Aerial Vehicles) is a natural consequence to overcome restrictions arising from stationary cameras. Going airborne offers the possibility to automatically obtain high resolution information on the concentrator geometry (using VIS cameras) and thermal losses (using IR cameras) for large fractions or even the entire solar field with virtually negligible impact on plant operation [1]. An approach to deduce mirror shape from airborne absorber reflection image is described in [2] and [3].

This article describes the application of several airborne optical measurement techniques to PTC (Parabolic Trough Collector) fields. It consists of a commercial UAV (Microdrones MD4-1000) and off-the-shelf cameras and auxiliary equipment. The central element is a Matlab based custom software toolbox for data-management, image processing and calculation of the concentrator geometry, with interfaces to photogrammetry and raytracing software. The target figures for geometric characterization of parabolic trough concentrators are:

- Mirror shape, described by slope deviations from the ideal parabola in curvature direction $\left(\mathrm{SD}_{\mathrm{X}}\right)$

- Absorber tube position, described by vertical and horizontal deviations from the focal line

- Tracking deviations, described by misalignment of the PTC optical axis and incoming radiation 
As standalone geometric quantities are not sufficient for yield analysis of the solar field, ray tracing software is applied to combine the measured geometric quantities with the sun shape and further assumptions ${ }^{2}$. The outcome of these simulations is the intercept factor, the ratio of radiation hitting the receiver and radiation available on the aperture. The target figure for thermal characterization [4] is essentially the surface temperature of the glass envelope tube of the HCEs (Absorber tube, aka: Heat Collecting Element) while the HTF temperature is close to operation temperature $\left(300-400^{\circ} \mathrm{C}\right)$. That way, defective and intact receivers can be distinguished. Beyond that, rough heat loss estimation may be possible by processing the measured temperatures with wind speed information in a thermal model [15]. The QFly measurement system presented hereinafter involves three measurement modes (see Figure 1), which differ mainly in measurement volume, flight altitude, spectral response of the camera and target figures. Table 1 provides an overview on the characteristics of these measurement modes.

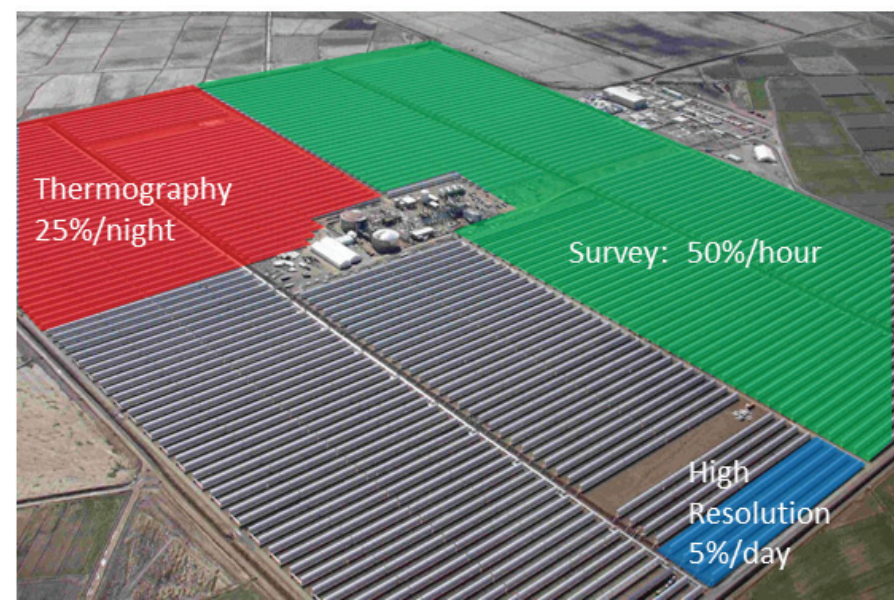

FIGURE 1: Typical effort for data acquisition for QFly measurement modes.

TABLE 1: Overview on characteristics of QFly measurement modes. Accuracy of survey results for mirror shape (*) and tracking accuracy $(* *)$ have been estimated, but not yet confirmed with independent measures.

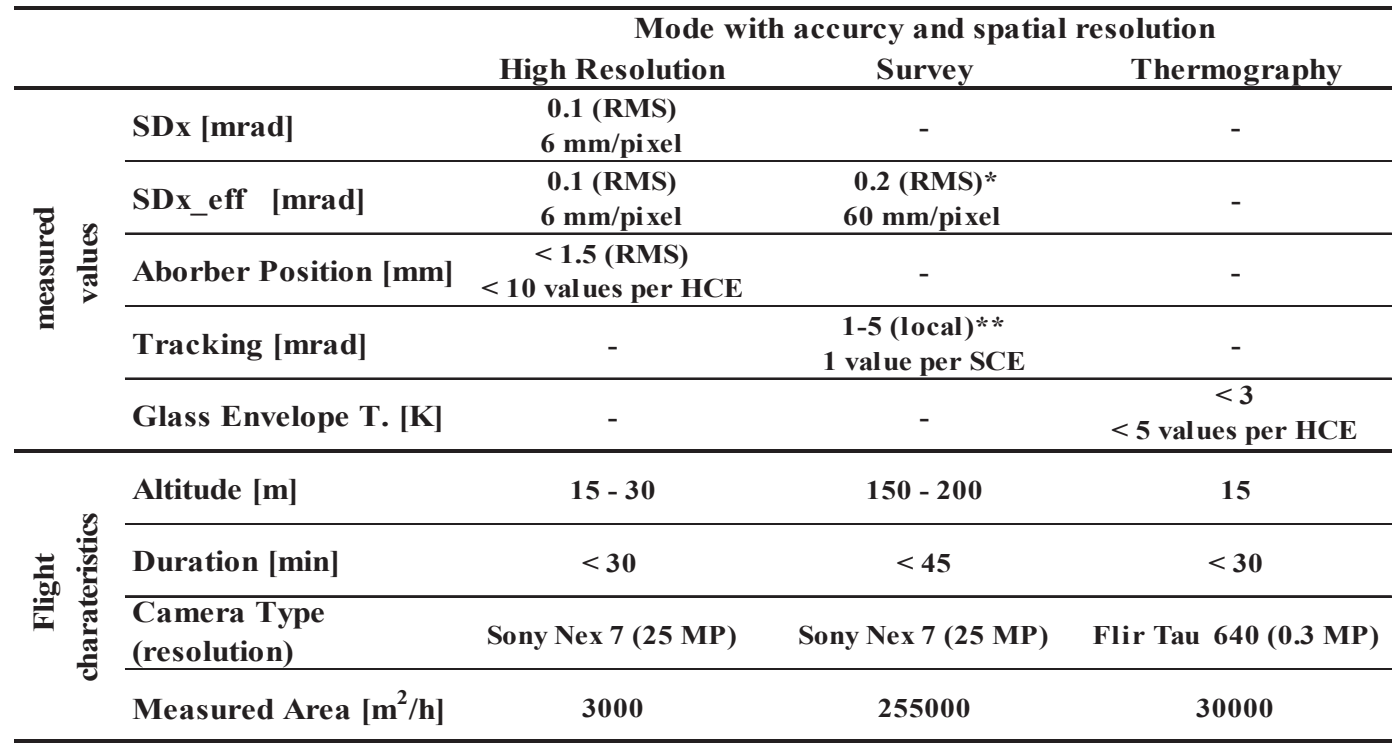

The accuracy of the results and the practicability of the airborne measurement depend on ambient condition like wind as well as soiling of the mirrors and glass envelope tube, respectively. Constant wind speeds higher than 6$8 \mathrm{~m} / \mathrm{s}$ prohibit the measurement due to safety considerations. Gusts could affect the image quality negatively. By 
very short exposure times and a pre-sorting of the images these influences can be compensated. Soiling or dew on the mirrors leads to weak contrast and makes the accurate detection of the absorber tube reflection much more difficult or even impossible. For this reason, cleaning the mirrors before the measurement is indispensable.

\section{HIGH RESOLUTION GEOMETRIC CHARACTERIZATION}

The high resolution airborne shape measurement approach and its validation have been presented in [5]. Here, a photogrammetric approach is applied to deduce the camera position from the images, as this is an essential parameter for the subsequent calculation of the mirror geometry based on the TARMES (Trough Absorber Reflection Measurement System) principle [6]. Developments beyond the concept presented in [5] comprise advanced image processing for absorber tube reflection- and feature-detection, the use of collector elements as photogrammetric features instead of application of artificial photogrammetric markers, and the implementation and validation of airborne absorber tube positioning [7] similar to an approach presented by NREL (National Renewable Energy Laboratory) [10].

\section{Advanced Image Processing}

The accurate detection of the absorber tube reflection is the basis for reliable prediction of mirror shape using the TARMES principle. Simple thresholding algorithms [8] deliver good results under ideal conditions. However, real conditions in the solar field may involve soiling on the mirror and inhomogeneous illumination, leading to weak contrast, noise, and bright stripes of direct sun reflections, causing erroneous detection of the absorber tube reflection (see Figure 2a). In order to cope with this, advanced segmentation methods have been combined with subtracting a background image created from the whole image series [9]. Additional measures are bilateral filtering and morphological operations. Ground truth data was created manually in order to measure the performance of the advanced image processing. The edge mismatch between detected and ground truth absorber tube reflection edge was used to assess the performance. The new methods show large improvements, allowing for complete automated image processing. In order to enhance the reliability and accuracy of the photogrammetric camera position relative to the collector, a large number of "natural" features of the mirror surface are detected and assigned by high-level image processing (see Figure 2b). For a typical ET (EuroTrough) parabolic trough module, 32 instead of only 4 edge reference points are available, which makes the camera positioning more robust in terms of the influence of possible outliers.

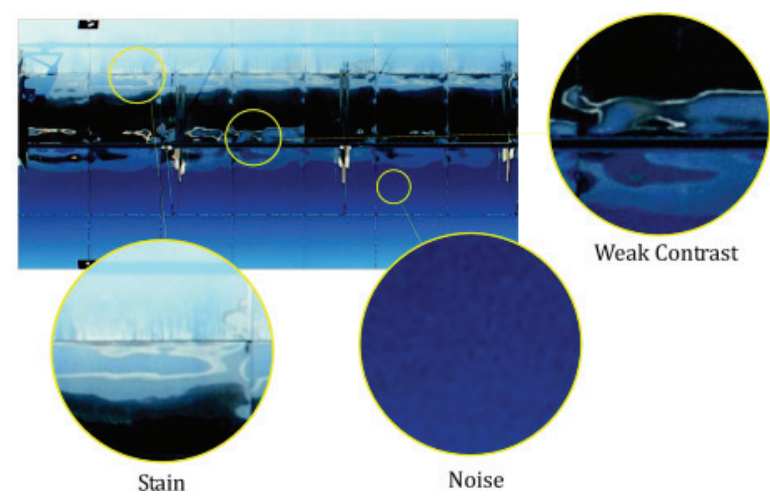

(a)

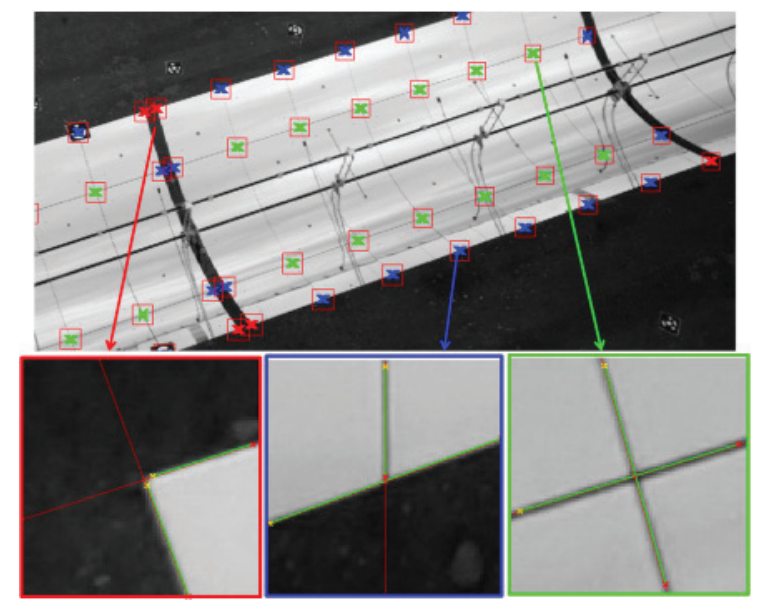

(b)

FIGURE 2a: Typical absorber tube reflection image with conditions that complicate proper detection of the absorber tube reflection. 2b: Feature of the mirror surface (gap, corners and crosses) are detected by image processing and serve as features for the photogrammetric evaluation. 


\section{Airborne Absorber Tube Positioning}

For two reasons, the absorber tube displacement along the optical axis and in lateral direction is of great importance for the qualification of parabolic trough collectors [10]. First, it is an indispensable parameter for the comprehensive ray-tracing analysis. Second, it is an input parameter for the deflectometric TARMES approach, which uses the reflection of the tube for the calculation of the mirror shape. The necessity for airborne absorber tube positioning arises from the desired degree of automation and lead to the development of a photogrammetric approach. The concept is to use the known camera positioning relative to each module to project a region of interest (ROI) perpendicular to the line of sight and centred on the design focal line. The centre line of the tube in the ROI is deduced from of the tube edges (see Figure 3a). The coordinates of the tube centre are calculated from at least two such observations with sufficient angular spacing in the XZ plane (see Figure $3 \mathrm{~b}$ ). In order to obtain sufficient measurement accuracy, in total 40-60 images are taken from an angular range of approx. $90^{\circ}$. The procedure is completely automated and delivers results with an accuracy in the range of $1.5 \mathrm{~mm}$ RMS. The accuracy has been validated at three ET SCEs (Solar Collector Elements) with $36 \mathrm{~m}$ of absorber tube using a manual close range photogrammetry benchmark of the glass envelope tube and semi-automatic detection of the absorber tube within the glass envelope tube. A detailed description of airborne absorber tube positioning will be available in [7].

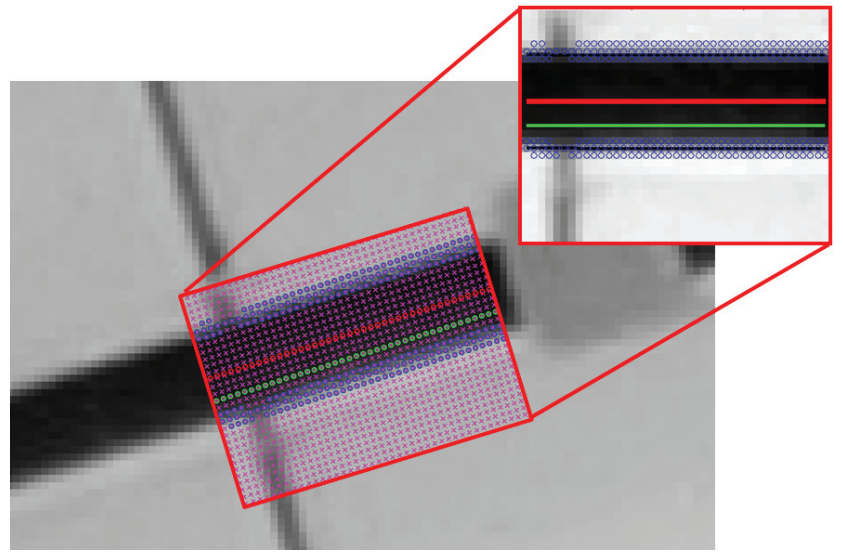

(a)

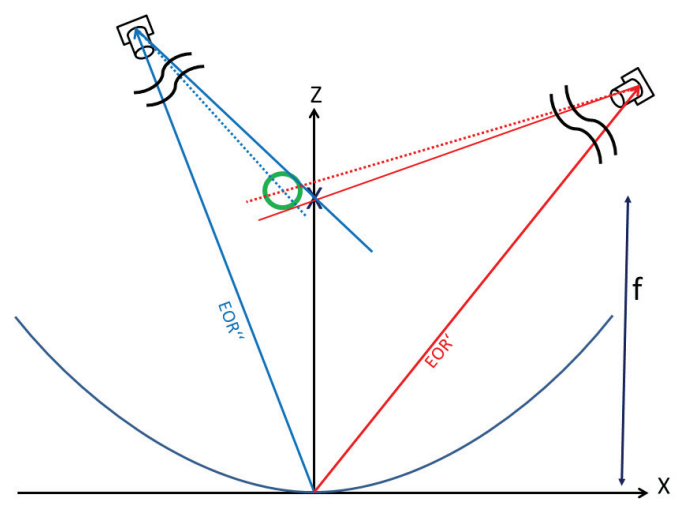

(b)

FIGURE 3a: Clipping with projected ROI (magenta crosses) and extracted ortho image. The design focal line (green) defines the center of the ortho image, while tube edges detected by image processing (blue) give the measured center line of the tube (red). 3b: Representation of the calculation of the tube position from two of normally up to 60 camera positions. The camera exterior orientations (EOR) relative to the parabola vertex are not presented proportionally. The LOS (line of sight) to the design focal line is represented with solid lines. Intersections of at least two LOS of the measured tube position (dotted) give the deviation from the focal line.

\section{SURVEY: A COMPLETE SOLAR FIELD SCAN}

Previously presented high resolution geometric characterization is employable for prototypes, single loops or fractions of the solar field. Plant operators however are, in the first place, interested in a global overview of the plant characteristics before having a closer look at certain areas. The survey approach combines the TARMES approach with images obtained from a flight height in the range of 150-200 $\mathrm{m}$ and delivers low resolution effective $\mathrm{s}^{3}$ slope deviations $\left(\mathrm{SDx}_{\mathrm{eff}}\right)$ and information on the actual orientation of the optical axis of each collector module.

Data is acquired by east-west flyovers with a horizontal velocity of approx. $3 \mathrm{~m} / \mathrm{s}$ and a frame rate of 2-3 fps. The main challenge is the robust and accurate determination of the camera position in a series of images showing repetitive patterns of the solar field from above. Fusion of image metadata and GNSS (Global Navigation Satellite System) position and orientation collected by the UAV leads to a robust approach to detect and trace the collector corners through the image series. A holistic camera model is used for off-line determination of the camera position and orientation, by projecting 3D solar field coordinates into the image and minimizing the difference of detected 
and projected 2D image coordinates. Typical residuals from that optimization of the camera position are $<1$ Pixel (RMS). It is necessary to distinguish between data acquired while the solar field is in operation and without concentrated radiation on the absorber tube. In the latter case, images were captured with a tracking offset of more than $5^{\circ}$ or under low DNI (Direct Normal Irradiance) conditions (see Figure 4a), which can be achieved at low sun elevation angles without impact on plant operation. With concentrated radiation on the absorber tube, there are areas with weak contrast between tube reflection and background (see Figure 4b), so that adaptions of the flight route are necessary to avoid areas with bright specular reflections.

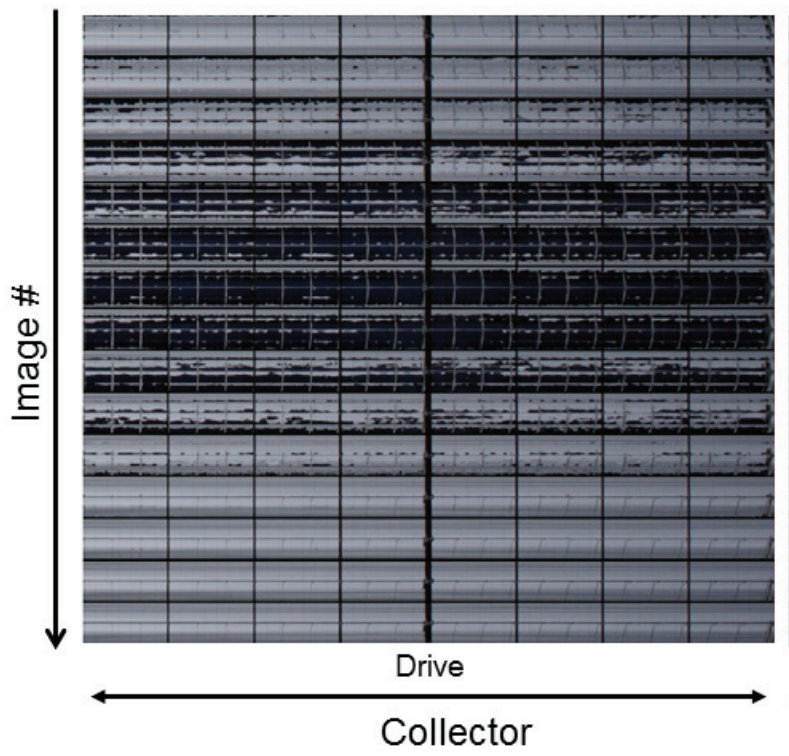

(a)

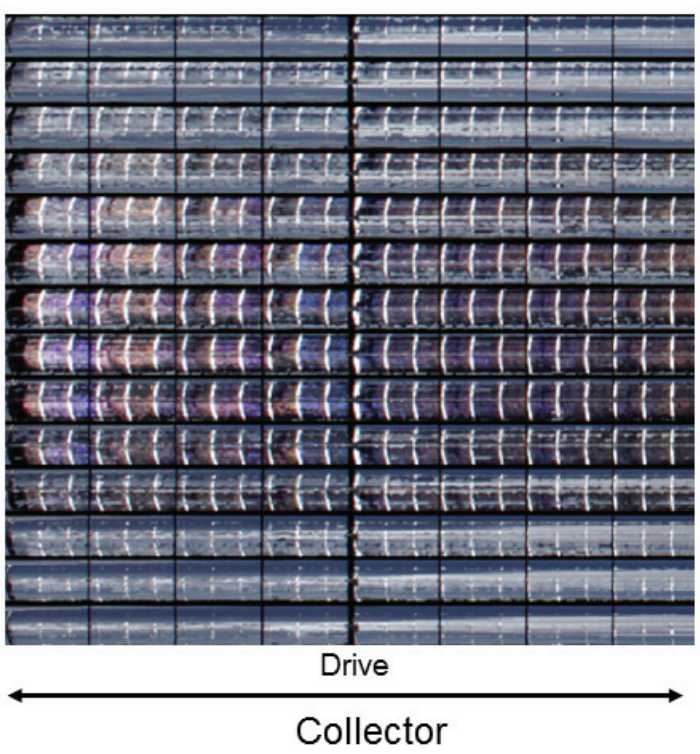

(b)

FIGURE 4a: Orthoimage series of a flight over a collector with $100 \mathrm{~m}$ east-west extension without concentrated radiation on the absorber. The good contrast allows for shape- and tracking measurements using the absorber tube reflection. 4b: An identical collector with concentrated radiation on the absorber tube. Bright areas on the left side complicate identification of the tube reflection, but the right side without direct reflections delivers a comparatively clear signal.

\section{Low Resolution $\mathbf{S D}_{\mathbf{X}}$}

The TARMES concept for shape measurements is identical for high (150-200 m) and low flight altitudes (15$30 \mathrm{~m}$ ). In the case of low flight altitudes, the smallest independently evaluated unit is an SCE (Solar Collector Element), while for the Survey mode entire SCAs (Solar Collector Assembly) are evaluated. Figure 5 shows SDx eff results for the same SCA/SCE obtained from both Survey and High Resolution mode and their intercomparison.

In addition to good qualitative matching of the maps, statistical value for the entire SCE and horizontal mirror rows are in good agreement. As expected, the lower sampling rate ${ }^{4}$ (15 instead of 30 images) of the presented Survey results tends to a slight underestimation of the RMS value. The expected uncertainty for the global SDx RMS value for the high resolution approach is in the range of $0.1 \mathrm{mrad}$ [5]. A detailed error analysis for the Survey method is not available yet, for the lack of a method to validate the camera positioning at this altitude. The good agreement between both approaches justifies limiting the camera position uncertainty (as the major contribution to SDx uncertainty) to a value below $100 \mathrm{~mm}$. The overall statement is that the survey approach is a promising method for fast and accurate scanning of entire PTC solar to determine the optical performance. 


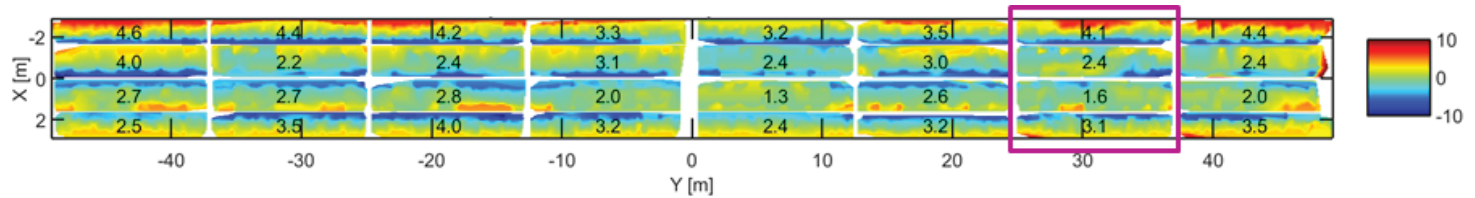

(a)

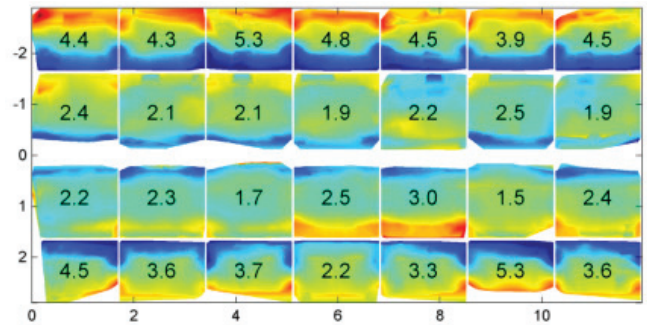

(b)

\begin{tabular}{c|c}
$\begin{array}{c}\text { Survey } \\
(\text { SDx RMS 2.9 mrad })\end{array}$ & $\begin{array}{c}\text { High Resolution } \\
\text { (SDx RMS 3.1 mrad })\end{array}$ \\
\hline 4,1 & 4,5 \\
\hline 2,4 & 2,1 \\
\hline 1,6 & 2,2 \\
\hline 3,1 & 3,8
\end{tabular}

(c)

FIGURE 5a: $\mathrm{SDx}_{\mathrm{eff}}$ in mrad for an entire SCA obtained from the Survey method with a spatial resolution of $50 \mathrm{~mm} / \mathrm{per}$ pixel from 15 images. 5b: $\mathrm{SDx}_{\mathrm{eff}}$ for the SCE highlighted above, with a spatial resolution of $6 \mathrm{~mm} /$ pixel from 30 images. 5c:

Comparison of global and row-wise SDx RMS values.

\section{Misalignment and Tracking}

An airborne approach offers the possibility to examine the tracking performance of the entire solar field. The particular aim for measuring tracking performance is the fact that tolerances for the alignment of each SCEs optical axis and the incoming radiation are in the range of $2 \mathrm{mrad}$ [12]. Determining factors for tracking accuracy are the performance of the drives system including sun-algorithm and (sun-) sensors and inclinometers, mounting accuracy (alignment between SCEs) and operational loads (wind, friction, and un-balance). So far, tracking performance was measured by manually mounted inclinometers on a random basis and is hardly accessible on a large scale. Series of airborne ortho-images as shown in Figure 4 and 6 reveal large potential for the tracking characterization, when combined with accurate information about the camera position [1].

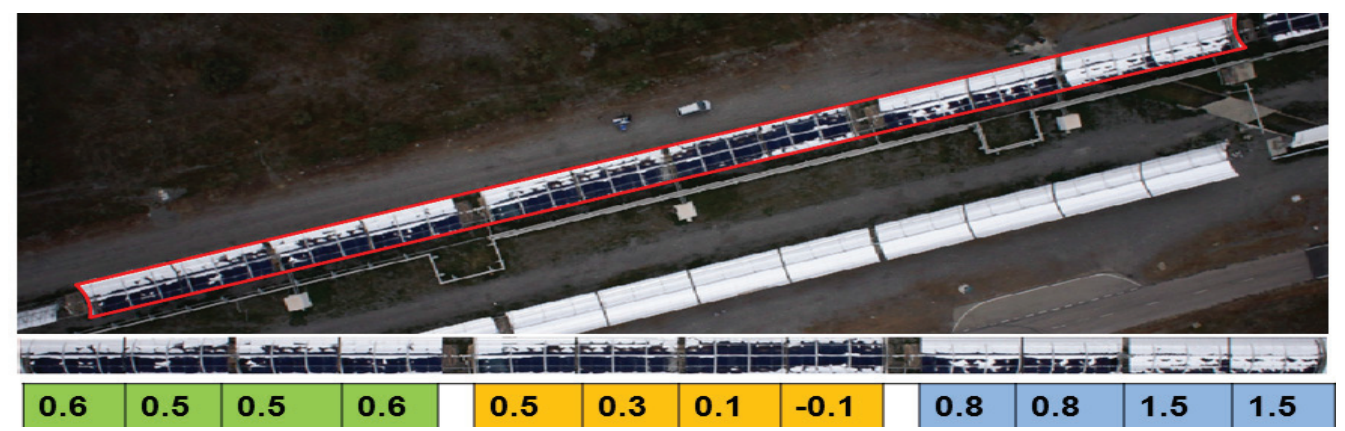

FIGURE 6: Aerial image of three SCAs, with corresponding ortho image and estimated relative module misalignment in [ $\left.{ }^{\circ}\right]$. The right part of the central SCA is oriented almost exactly towards the camera, while the left and the right show pronounced deviations. Possible reasons for the observed deviations are offsets of the tracking system, friction and misalignment between SCEs.

For the validation of these results, it is necessary to repeat the airborne data acquisition while high-precision inclinometers are observing the absolute orientation. Rough error estimations predict an accuracy of approx. $1 \mathrm{mrad}$, while the main contribution is expected to originate from the camera position uncertainty. 


\section{AIRBORNE THERMOGRAPHY}

Minimizing heat losses from the absorber tubes is a major concern for HCE manufacturers and plant operators. Vacuum loss and $\mathrm{H}_{2}$ penetration into the vacuum annulus are main threats for thermal plant performance [11]. Thermography of the glass envelope tube delivers reliable indications on the insulation properties [4] [13] and has also been proposed for airborne data acquisition [1]. We implemented a straightforward approach to assess glass envelope temperatures from airborne IR images by using a FLIR T-640 and by data fusion of image metadata and GNSS and IMU (Inertial Measurement Unit) information from the flight recorder [14]. Image based glass envelope temperatures have been cross checked with contact measurements with resistance thermometers. The uncertainty analysis reveals a measurement error of the glass envelope temperature from IR images with the current set-up in the range $<3^{\circ} \mathrm{K}$. Heat loss can be estimated approximately by processing the measured glass envelope tube temperatures together with wind and further ambient information in a thermal model as the one proposed by SunLab (Sandia \& NREL) in [15]. The airborne IR system has been developed and validated on SCE scale. Combining the frame rate of the camera with typical cruise velocity $(5 \mathrm{~m} / \mathrm{s})$ and flight duration ( $>30 \mathrm{~min})$ of the UAV, up to $9 \mathrm{~km}$ of absorber tubes can be scanned per flight, allowing for screening of at least a quarter of a typical $50 \mathrm{MW}_{\mathrm{el}}$ PTC solar field per night.

\section{METHODOLOGY FOR PLANT OPTIMIZATION}

The objective of airborne PTC field characterization is providing a comprehensive tool to solar plant operators, owners, and EPC (Engineering, Procurement and Construction) contractors. While qualification of collector modules and components should be performed in the production processes, the QFly system measures the performance of a solar field after its completion. This offers the possibility to define performance parameters for an approval of the optical quality of the entire solar field.

In an operational power plant, the system allows identifying low-performing areas, with the objective to optimize the solar field performance with suited correction measures. Plant optimization involves the participation of the operator to assess costs and benefits, and to implement the optimization measures (see Figure 7). Even small improvements of the solar field performance in the range of $1 \%$ result in a financial gain over the operational life in the range of tens of millions of Euros ${ }^{5}$. It must be stated that it is challenging to adjust losses arising from exceeding the tight tolerances for mirror- and receiver geometry. More possibilities are viable for optimizing the alignment and tracking procedure, and for targeted replacement of absorber tubes [11].

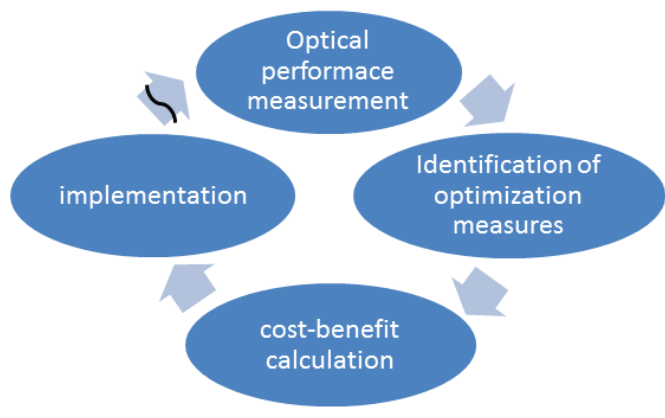

FIGURE 7: Methodology for plant optimization. After each implementation, iteration might be required to reach the optimum.

\section{SUMMARY AND OUTLOOK}

DLR has developed a comprehensive tool for airborne optical characterization of parabolic trough solar fields. Results for collector geometry can be acquired with different spatial resolutions and recording speeds. All geometric measures have been cross-checked with independent measures or already validated techniques; except the newly developed airborne tracking characterization. In order to validate the tracking characterization, new data will be 
acquired while logging the actual orientation of selected collectors with high precision inclinometers. IR based screening for defective absorber tubes has proven to deliver reliable results at SCE level. For the airborne thermography, data acquisition and post-processing will be upscaled to complete solar fields in the near future.

There are concepts under development to transfer the skills and tools from airborne PTC characterization to the optimization of heliostat fields. The motivation is here to achieve substantial time saving by airborne tracking characterization instead of methods based on flux density measurements of single heliostats.

\section{REFERENCES}

1. JORGENSEN, G., et al. Assess the Efficacy of an Aerial Distant Observer Tool Capable of Rapid Analysis of Large Sections of Collector Fields, FY 2008 CSP Milestone Report, NREL/MP-550-44332.

2. STYNES, J. K., and IHAS, B. Slope Error Measurement Tool for Solar Parabolic Trough Collectors. National Renewable Energy Laboratory (NREL), Golden, CO., 2012.

3. OWKES, J. K. An Optical Characterization Technique for Parabolic Trough Solar Collectors Using Images of the Absorber Reflection. Doctoral thesis submitted to the University of Colorado, 2012

4. PRICE, H., et al. Field survey of parabolic trough receiver thermal performance. In: ASME 2006 International Solar Energy Conference. American Society of Mechanical Engineers, 2006. S. 109-116.

5. PRAHL, C., et al. Airborne shape measurement of parabolic trough collector fields. Solar Energy, 2013, 91. Jg., S. 68-78.

6. ULMER, S., et al. Slope error measurements of parabolic troughs using the reflected image of the absorber tube. Journal of Solar Energy Engineering, 2009, 131. Jg., Nr. 1, S. 011014.

7. PRAHL, C., et al. Absorber Tube Displacement in Parabolic Trough Collectors - A review and presentation of an airborne measurement approach. Manuscript submitted to Solar Energy

8. Otsu, N., "A Threshold Selection Method from Gray-Level Histograms," IEEE Transactions on Systems, Man, and Cybernetics, Vol. 9, No. 1, 1979, pp. 62-66.

9. HERTEL, J. Study and Implementation of an Object Tracing Algorithm for the Deflectrometric Shape Qualification of Parabolic Trough Collectors. 2013. Master Thesis. Technical University of Munich.

10. STYNES, J. K., and IHAS, B. Absorber Alignment Measurement Tool for Solar Parabolic Trough collectors. ASME 2012 6th International Conference on Energy Sustainability. 2012.

11. RÖGER, M., et al. Techno-economic analysis of receiver replacement scenarios in a parabolic trough field. In: SolarPACES 2015 Conference, Cape Town, South Africa. 2015. S. 13-16. .

12. POTTLER, K., et al. Ensuring performance by geometric quality control and specifications for parabolic trough solar fields. Energy Procedia, 2014, 49. Jg., S. 2170-2179.

13. CARON, S.; RÖGER, M. In-situ heat loss measurements of parabolic trough receivers based on transient infrared thermography. Solar Energy, 2016, 135. Jg., S. 111-121.

14. NATHO, R.. Thermal Evaluation of Receivers in Parabolic Trough Collectors using Airborne Infrared Imaging. 2012. Bachelorthesis. Ilmenau University of Technology.

15. PRICE, H., et al. 2006. Field survey of parabolic trough receiver thermal performance. In ASME 2006 International Solar Energy Conference (pp. 109-116). American Society of Mechanical Engineers. 